\section{BASIC programs for observational research using the TRS-80 Model 100 portable and Model 4 computers}

\author{
RICHARD DENI, KATHERINE SZIJARTO, \\ ALANE EISLER, and CHRISTINA FANTAUZZO \\ Rider College, Lawrenceville, New Jersey
}

Portable computers have the potential for widespread use in behavioral research. The programs described here were written to allow the TRS- 80 Model 100 portable computer to record and process data from behavioral observations and to transfer those data to a larger system, the Model 4, for storage on flexible disks and further data analysis.

A number of specially designed electronic data recorders have been in use for some time. Their cost, however, has been substantial, and their flexibility limited, when compared with general-purpose microcomputers. Portable computers, such as the TRS- 80 Model 100, offer the observational researcher a mix of flexibility and power to record, store, and reduce streams of coded behavior. By using the Model 100, the researcher has full portability, a typewriter keyboard, a text editor, extended BASIC, up to $32 \mathrm{~K}$ RAM, RAM file storage, off-line storage on cassette tape, and data communications capabilities. This portable computer can serve in several capacities beyond its use to record, store, and reduce coded behaviors during an observational session. Two programs for the entry and management of streams of coded behaviors are described below.

\section{Description of Programs}

RECORD Program. RECORD is a program for the Model 100 that is used during the actual observation session. Single keystrokes can be assigned as behavioral codes. Two types of codes are possible. Timed codes consist of a group of keyboard characters that make up a set of mutually exclusive event states. By definition, only one of these states may be in effect at any time. The cumulative duration (to the nearest second) of each state may be computed from the stream of codes. Timed codes are also counted. The second type is a simple counted code. A cumulative count of these codes in the stream may be computed. The timing of a state does not affect the counting of any codes occurring during the interval.

When the observer starts the RECORD program, a large array that will store each character code as it is entered, along with the clock time at entry (in whole seconds) is dimensioned. The observer may stop the session by a keyboard entry or may preset the program to stop after a certain amount of clock time has elapsed.

The authors' mailing address is: Department of Psychology, Rider College, Lawrenceville, New Jersey 08648.
Once the session has completed, RECORD opens a RAM file in the Model 100 and transfers the code-time stream to memory under the file name specified by the observer. The data, now in a RAM file, can be transferred to a cassette tape for permanent off-line storage and input to the Model 4.

Input of Cassette Files to Model 4. The Model 4 utility program TAPE100 (supplied with the diskoperating system) is used to read cassette files produced by the RECORD program. TAPE 100 puts the data file on disk under a file name supplied by the user. Cassette tapes may be retained as backup copies of raw data or may be reused following bulk erasure.

REDUCE Program. The next step in the management of data files is running the REDUCE program. This program summarizes the observational code streams by printing the frequency and duration of codes, and the results of sequential analyses. Two types of sequential analysis are available: cumulative sequence frequency and duration. Any code sequence may be specified. Sequence durations can show how long a given sequence was in effect or how long it took for the completion of a given sequence. The above analyses include automatic printing of summary tables through the Model 4 Centronics-compatible parallel port. The original data files remain stored on disk and may be backed up by system copy utilities.

The REDUCE program contains other options for the listing of data disk directories, the printing of raw data streams, and the removal of data files from the data disk. REDUCE should be configured separately for each specific observation procedure. A series of DATA statements can be edited to supply REDUCE with timed and counted codes, and code sequences to be analyzed.

\section{Transportability}

These programs were written for the MICROSOFT BASIC that is standard on the TRS- 80 Model 100 and Model 4; however, they will run on any comparable BASIC that has access to a time-of-day clock. In addition, the RECORD program can be easily adapted to run on a desktop computer if portability is not required. The equipment requirement for the programs is as follows: TRS- 80 Model 100 computer, 32K expansion, cassette recorder, ac adapter, leaderless cassette tapes; TRS-80 Model 4 computer, two disk drives, cassette recorder, and flexible disks.

\section{Availability}

Program listings may be obtained at no charge from Richard Deni, Psychology Department, Rider College, Lawrenceville, New Jersey 08648.

(Manuscript accepted for publication December 2, 1983.) 